\title{
Comparison of urinary KIM-1 and NGAL and plasma creatinine in patients undergoing coronary artery bypass graft surgery
}

\author{
Maryam Saeedi Ghaheh $^{1}{ }^{\circledR}$, Saeed Mardani $^{{ }^{*} \odot}$, Afsaneh Malekpour ${ }^{\circledR}{ }^{\circledR}$, Farzaneh Kadkhodaei

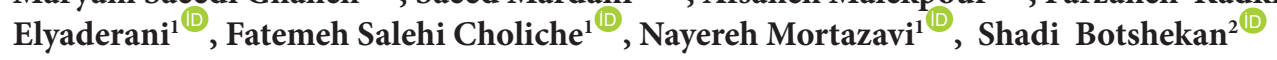 \\ ${ }^{1}$ Department of Internal Medicine, Faculty of Medicine, Shahrekord University of Medical Sciences, Shahrekord, Iran \\ ${ }^{2}$ Faculty of Medicine, Shahrekord University of Medical Sciences, Shahrekord, Iran
}

\section{A R T I C L E I N F O}

\section{Article Type:}

Original

\section{Article History:}

Received: 19 March 2020

Accepted: 8 June 2020

Published online: 25 June 2020

\section{Keywords:}

Neutrophil gelatinase-associated lipocalin (NGAL), Acute renal injury, Coronary artery bypass graft surgery, Kidney injury molecule (KIM-1)

\begin{abstract}
A B S T R A C T
Introduction: Serum creatinine level is currently being used as an indicator to detect acute kidney injury (AKI) after cardiac surgery, which is delayed and unreliable.

Objectives: This study was conducted to determine the AKI in patients undergoing coronary artery bypass graft $(\mathrm{CABG})$ surgery by measurement of urinary creatinine and plasma kidney injury molecule (KIM-1) and neutrophil gelatinase-associated lipocalin (NGAL).

Patients and Methods: In this cross-sectional study, 96 patients undergoing CABG were divided based on their serum creatinine level of the fourth day after procedure into two groups of AKI (an increase of creatinine more than $0.3 \mathrm{mg} / \mathrm{dl}$ or more than $150 \%$ of its baseline level) and non-AKI. In both groups urinary KIM-1 and urinary NGAL were measured on the first day of surgery (first $24 \mathrm{~h})$.

Results: No significant difference was observed in baseline creatinine between the two groups $(P>0.05)$. However postoperative serum creatinine, creatinine changes, urinary NGAL and KIM1 showed significant difference between the groups $(P<0.05)$. Serum creatinine was positively correlated with KIM-1 $(\mathrm{r}=0.666, P<0.001)$ and NGAL $(\mathrm{r}=0.660, P<0.001)$. KIM-1 and NGAL had high ability to detect AKI [area under the ROC (receiver operating characteristic) curve of 0.929 and 0.838 , respectively]. The NGAL at cut-off point of 97.4 had $86 \%$ sensitivity and $80 \%$ specificity and KIM-1 at cut-off point of 14.8 had $84 \%$ sensitivity and $89 \%$ specificity.

Conclusion: KIM-1 and NGAL had high ability to detect AKI. However, KIM-1 had higher detection ability than that of NGAL.
\end{abstract}

\section{Implication for health policy/practice/research/medical education:}

Acute kidney injury (AKI) is a common and serious condition, its diagnosis depends on serum creatinine, which is a delayed and unreliable indicator of AKI. In this study, postoperative creatinine, creatinine changes, urinary neutrophil gelatinase-associated lipocalin (NGAL) and urinary kidney injury molecule (KIM-1) were significantly higher in AKI patients than non-AKI patients. Serum creatinine was positively correlated with KIM-1 and NGAL. KIM-1 and NGAL had high ability to detect AKI. The availability of a suitable AKI biomarker could further revolutionize renal and critical care. However, further studies are needed for identification, validation, commercial development and acceptance of developed AKI biomarkers.

Please cite this paper as: Saeedi Ghaheh M, Mardani S, Malekpour A, Kadkhodaei Elyaderani F, Salehi Choliche F, Mortazavi $\mathrm{N}$, Botshekan S. Comparison of urinary KIM-1 and NGAL and plasma creatinine in patients undergoing coronary artery bypass graft surgery. J Nephropharmacol. 2021;10(1):eo4. DOI: 10.34172/npj.2021.04.

\section{Introduction}

Acute kidney injury (AKI) is one of the main complications observed after coronary artery bypass graft $(\mathrm{CABG})$ surgery and re-vascularization of coronary arteries through the skin or percutaneous coronary intervention (PCI) with a prevalence of about $1 \%-30 \%$ depending on the study population (1). Higher age and the history of chronic kidney disease (CKD), are common risk factors for AKI in CABG and PCI. However, there are specific factors in any type of re-vascularization, for example, using cardiopulmonary bypass in $\mathrm{CABG}$ and contrast nephropathy in PCI which are the specific risk factors of AKI (2-4). In patients with AKI, not only the hospitalization stay and consequent postoperative complications increase, but also the risks for long-term side effects including CKD and end-stage renal disease 
(ESRD) may increase (5).

In AKI, early diagnosis is an essential key for successful treatment. Serum creatinine is currently used to diagnose renal dysfunction after surgery. However serum creatinine may not increase until a few days after the tubular injury, which results in a delay in the diagnosis and treatment of kidney damage $(6,7)$. Therefore, the development of new AKI biomarkers is of great necessity for clinical and research purposes. An ideal biomarker should be non-invasive, fast and easy to perform and have a high sensitivity and specificity (8).

Neutrophil gelatinase-associated lipocalin (NGAL), also known as lipocalin 2 (LCN2) and oncogenes $24 \mathrm{p} 3$, is a 25$\mathrm{kDa}$ protein bound to neutrophils which is produced by neutrophils and a variety of epithelial cells. It is also found in variable levels in the stomach, colon, kidney, trachea, lung, uterus, prostate and salivary glands (9). In AKI, NGAL is excreted in large amounts in the blood and urine within two hours post-injury. NGAL levels in patients with AKI is associated with the severity and prognosis of the disease and can be used as an indicator for AKI (10). In this regard, clinical studies have shown that elevated NGAL levels have a high sensitivity and specificity for predicting AKI after surgery, especially in the first two hours after pediatric heart surgery (11). Previously, a significant relationship between increased urinary NGAL and AKI in adult patients undergoing heart surgery was shown (12).

Hepatitis A virus cellular receptor 1 (HAVcr-1), also known as T-cell immunoglobulin, mucin domain 1 (TIM-1) and kidney injury molecule-1 (KIM-1), plays an important role in regulating cellular immunity, especially in host response to the viral infection. The KIM-1 or TIM1 marker is secreted from proximal tubular epithelial cells during ischemia and reduced blood flow to the kidney. This biomarker is secreted during the first 24-48 hours of insults which induced cell destruction, thereby it can be used as a primary biomarker in the diagnosis of AKI after heart surgery (13). Parikh et al showed that KIM-1 can be considered as an independent biomarker to indicate AKI after cardiac surgery and its associated side effects (14).

\section{Objectives}

The present study was aimed to determine urinary and plasma biomarkers in patients undergoing coronary artery bypass surgery and also to determine their diagnostic value.

\section{Patients and Methods}

\section{Study design}

This study was a cross-sectional descriptive-analytical, conducted on 96 patients aged between 40 to 65 years who referred for CABG surgery to the hospitals of Shahrekord University of Medical Science, Shahrekord, Iran (2018). Patients with high risk obesity (body mass index; $\mathrm{BMI} \geq 40 \mathrm{~kg} / \mathrm{m}^{2}$ ), CKD, liver failure, drug abusers and coagulation problems were not included in the study. Additionally, patients who underwent heart-lung surgery without using a cardiopulmonary bypass pump or underwent simultaneous heart value and CABG surgeries were excluded from the study. The method of induction of anesthesia was the same in all patients and was based on the use of narcotic drugs and benzodiazepines as well as the use of muscle relaxants. To maintain anesthesia isoflurane and oxygen were used. Patient information such as age, gender, weight, height, risk factors including high blood pressure, diabetes mellitus, high fat, smoking and a positive family history of coronary artery disease were recorded in a special form. Before the procedure or surgery and after taking the signed consent form, patients' serum creatinine level was measured to obtain its baseline level. Patients' serum creatinine level was measured again after 4 days of surgery, then the individuals were divided into two groups based on the emerging situation of acute kidney injury; AKI (increasing $0.3 \mathrm{mg} / \mathrm{dL}$ from baseline creatinine or more than 150\%) and non-AKI. In both groups, urinary levels of KIM-1 and NGAL, were measured on the first day of surgery (first 24 hours after surgery) too.

\section{Ethical issues}

The research followed the tenets of the Declaration of Helsinki. The Ethics Committee of Shahrekord University of Medical Sciences approved this study. The institutional ethical committee at Shahrekord University of Medical Sciences approved all study protocols (IR.SKUMS.MSP. REC.1397.176). Accordingly, written informed consent taken from all participants before any intervention. This study was the result of the internal medicine residential thesis of Maryam Saeedi at this university.

\section{Data analysis}

Data were analyzed by SPSS software. To describe the data, frequency indices and percentage were used for qualitative variables and mean \pm standard deviation were used for quantitative variables. To test the differences between the studied groups, the independent $t$ test (for approved data normality), Mann-Whitney U test (for rejected data normality) and chi-square test were used. The relationship between quantitative variables with normal distribution was determined using Pearson's correlation coefficient since the Spearman's correlation coefficient was used for qualitative variables or quantitative variables with no normal distribution. Moreover, ROC (receiver operating characteristic) curve was used for determining cutting point of the tests. In all tests, the significance level was considered at $P<0.05$.

\section{Results}

In the present cross-sectional study, 96 patients undergoing CABG surgery were divided into two groups of AKI (45 people, $46.9 \%$ ) and non-AKI (51 people, 53.1\%) patients 
according to the serum creatinine levels of the fourth day after operation. The non-AKI group included 39 men (76.5\%) and 12 women (23.5\%) while the AKI group included 29 men $(64.4 \%)$ and 16 women (35.6\%). The two groups did not show any significant difference in terms of gender distribution $(P>0.05)$. Additionally, the mean age of patients in the AKI (50.04 \pm 6.02 years) and nonAKI (48.88 \pm 4.52 years) groups did not show significant difference $(P>0.05)$. However, a significant difference was observed regarding BMI between two groups of AKI and non-AKI $\left(30.25 \pm 25.13 \mathrm{~kg} / \mathrm{m}^{2}\right.$ versus $23.91 \pm 2.40 \mathrm{~kg} /$ $\mathrm{m}^{2}$ respectively; $\left.P<0.05\right)$.

As shown in Table 1, the baseline serum creatinine was not significantly different between AKI and non-AKI groups $(P>0.05)$, however the post-operative creatinine, NGAL and KIM-1 were significantly higher in AKI group compared with those of non-AKI group $(P<0.05)$.

As shown in Table 2, serum creatinine of all patients had significantly positive relationship with KIM-1 $(\mathrm{r}=0.666$, $P<0.001)$ and NGAL $(\mathrm{r}=0.66, P<0.001)$. Furthermore, in patients with AKI, serum creatinine had significant positive correlations with KAM-1 $(\mathrm{r}=0.448, P<0.001)$ and NGAL $(\mathrm{r}=0.544, P<0.001)$. In contrast; in non-AKI patients, serum creatinine was not significantly correlated with KIM-1 or NGAL $(P>0.05)$.

Table 1. Comparison of serum creatinine, NGAL and KIM-1 between AKI and non-AKI groups

\begin{tabular}{llll}
\hline Variable & $\begin{array}{l}\text { Non-AKI group } \\
\text { Mean } \pm \text { SD }\end{array}$ & $\begin{array}{l}\text { AKI group } \\
\text { Mean } \pm \text { SD }\end{array}$ & $P$ value \\
\hline $\begin{array}{l}\text { Baseline creatinine } \\
(\mathrm{mg} / \mathrm{dL})\end{array}$ & $0.99 \pm 0.24$ & $1.04 \pm 0.27$ & 0.335 \\
$\begin{array}{l}\text { Post-operative } \\
\text { creatinine }(\mathrm{mg} / \mathrm{dL})\end{array}$ & $1.05 \pm 0.24$ & $1.82 \pm 0.64$ & $<0.001$ \\
$\begin{array}{l}\text { Creatinine level } \\
\text { change }(\mathrm{mg} / \mathrm{dL})\end{array}$ & $0.059 \pm 0.16$ & $0.78 \pm 0.57$ & $<0.001$ \\
$\begin{array}{l}\text { Urinary NGAL }(\mathrm{ng} / \mathrm{dL}) \\
\text { Urinary KIM-1 }(\mathrm{ng} / \mathrm{dL})\end{array}$ & $19.54 \pm 31.47$ & $171.14 \pm 173.63$ & $<0.001$ \\
\hline
\end{tabular}

Table 2. The relationship between KIM-1 and NGAL based on spearman correlation test

\begin{tabular}{lcc}
\hline Group & KIM-1 & NGAL \\
\hline All patients $(n=96)$ & $R=0.666$ & $R=0.660$ \\
Non-AKI $(n=61)$ & $P<0.001 * *$ & $P<0.001 * *$ \\
AKI $(n=45)$ & $R=0.097$ & $R=0.232$ \\
& $P=0.499$ & $P=0.101$ \\
\hline
\end{tabular}

${ }^{* *}$ The significant difference at $P<0.001$ level.

Table 3. The area under ROC for KIM-1 and NGAL factors

\begin{tabular}{lccc}
\hline Factor & The area under ROC curve & SE & $95 \% \mathbf{C l}$ \\
\hline KIM-1 & 0.929 & 0.026 & $0.879-0.979$ \\
NGAL & 0.838 & 0.035 & $0.814-0.952$ \\
\hline
\end{tabular}

$\mathrm{SE}$, standard error; $\mathrm{Cl}$, confidence interval.
As shown also in Table 3, the area under the ROC curve of the KIM-1 and NGAL factors, which indicate their diagnostic power was 0.929 and 0.838 respectively. The results indicated the high diagnostic power of both factors, although the KIM-1 showed greater diagnostic power compared to NGAL (based on a contractual classification system, the area under the curve of $0.9-1$ indicates the great power while area under the curve of 0.8-0.9 indicates good power). For NGAL at the cutting point of $97.4 \mathrm{ng} /$ $\mathrm{dL}$ the sensitivity of $86 \%$ and the specificity of $80 \%$ were observed while the sensitivity of $84 \%$ and specificity of $89 \%$ were observed for the KIM-1 at cutting point of 14.8 ng/dL.

\section{Discussion}

In the present cross-sectional study, 96 patients who underwent $\mathrm{CABG}$ surgery were divided into two groups of AKI and non- AKI (based on the post-operating serum creatinine of day forth). The two groups did not show any significant difference regarding gender and age distribution $(P>0.05)$. Moreover, BMI level was higher in AKI group $\left(30.25 \pm 25.13 \mathrm{~kg} / \mathrm{m}^{2}\right)$ than that of non-AKI group $\left(23.91 \pm 2.40 \mathrm{~kg} / \mathrm{m}^{2} ; P<0.05\right)$. Zou et al reported the risk of AKI in individuals with underweight, normal weight, overweight and obese who underwent cardiac surgery was respectively $29.9 \%, 31 \%, 36.5 \%$ and $46 \%$, which showed a significant difference $(P<0.05)$ among them (15). Khreba et al reported that the mean age of patients who underwent CABG with AKI and without AKI were respectively 47.40 and 44.55 years. However, the difference was not significant (16). Likewise, Han et al studied the prevalence of AKI in patients who underwent $\mathrm{CABG}$ and found, patients with AKI were more likely to be older (17).

In our study, the baseline creatinine was not significantly different between AKI and non-AKI groups $(P>0.05)$. Additionally, the post-operative serum creatinine was significantly higher in AKI group compared with that of non-AKI group $(P<0.05)$. Recently in the study conducted by Khreba et al, a significant increase in serum creatinine in AKI group 24 hours after surgery compared to that of non-AKI group was observed. None of their patients with AKI showed an absolute increase greater than $0.3 \mathrm{mg} / \mathrm{dL}$ in serum creatinine at baseline in the early hours after surgery (16). Han et al (17) and Mishra et al (11) also concluded that changes in serum creatinine were delayed, typically occurring 24 to 48 hours after the start of surgery.

In our study, the area below the ROC curve of urinary KIM-1 factor was 0.929 , indicating its high diagnostic power in identifying AKI. For KIM-1 at the cutting point of $14.8 \mathrm{ng} / \mathrm{dL}$, the sensitivity of $84 \%$ and the specificity of $89 \%$ were observed. Torregrosa et al showed the area below ROC curve for KIM-1 was 0.713 in patients under angiography and 0.716 in patients undergoing 

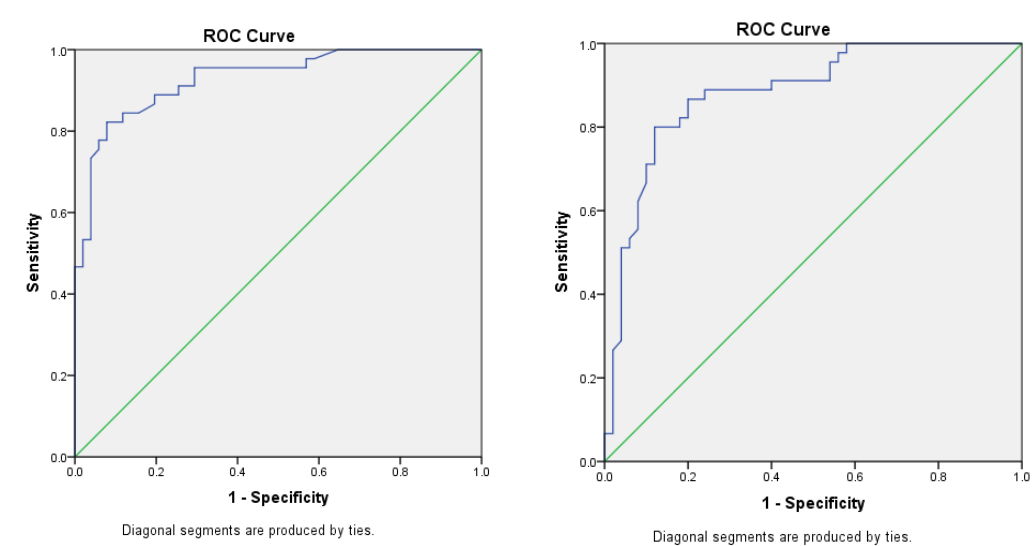

Figure 1. ROC curve of KIM-1 (A) and NGAL (B).

cardiac surgery. In their study, the urinary KIM-I level was determined 12 hours after surgery (13). Recently, in the study by Khreba et al, the area under the ROC curve of the KIM-1 biomarker in patients who underwent CABG was 0.751 . At the cutting point of 1.9 , the KIM-1 sensitivity for AKI detection was $48 \%$ and its specification was $94 \%$. They measured the KIM-1 three hours after surgery (16). Previously, Han et al observed that the area below ROC for KIM-1 to predict AKI immediately and three hours after surgery was 0.68 and 0.65 , respectively (17). Previously, Du et al studied a group of 252 children referring to emergency unit and found that $7.1 \%$ of them had AKI since the predicting value of the KIM-1 for AKI was 0.73 (18). The differences appear to be most likely related to patient's heterogeneity, the small populations under study and the difference in the post-operative sampling time. Additionally, the urinary concentration of KIM-1 may be affected by several factors such as sepsis, the administration of contrast medium and some common diseases including atherosclerosis, hypertension and diabetes (17). Our study compared to other studies showed a higher predicting value of KIM-1 to diagnose AKI, which is probably due to the measurement of KIM-1 levels 24 hours after surgery.

Our study showed also that the area below the ROC curve for plasma NGAL, which indicating its diagnostic power was 0.838 which reveals its high diagnostic power. NGAL at the cutting point of 97.4 had the sensitivity of $86 \%$ and a specificity of $80 \%$. Torregrosa et al reported that the area under the ROC curve for urinary NGAL was 0.958 in patients under angiography and 0.916 in patients undergoing cardiac surgery while in their study NGAL levels in patients' urine samples were determined 12 hours after surgery (13). In a study by Parikh et al after multivariate adjustment, plasma NGAL which exceeded the fifth percentile was associated with 5-fold higher of AKI. The area below the ROC curve for NGAL was 0.750 in patients with open heart surgery after six hours of surgery. Moreover, plasma NGAL had more predicting value than serum NGAL (19). In a previous meta-analysis conducted by Haase et al, the area below the ROC curve for the NGAL factor was 0.775 for cardiac surgery and 0.894 for coronary angiography (20). Koyner et al reported the area below the ROC curve for plasma and urinary NGAL at 6 hours after surgery was 0.536 and 0.691 respectively (21). Accordingly in the study by Wagener et al, the area below the ROC curve for urinary NGAL factor was 0.573 , $0.603,0.611$ and 0.584 immediately after surgery, at 3 , at 18 and 24 hours after surgery respectively (8). In another study in 2009 , the area below the ROC curve for plasma and urinary NGAL was 0.80 and 0.96 (22). The differences between the values reported in the different studies are due to the differences in sampling time, sample type and population studies.

In our study the area under the ROC curve of the KIM1 and NGAL factors which indicate their diagnostic power was 0.929 and 0.838 respectively. The results indicated the high diagnostic power of both factors although the KIM1 showed greater diagnostic power compared to NGAL. Han et al found that the area below the ROC curve for the KIM-1 factor to predict AKI was higher than NGAL immediately and three hours postoperatively (0.680 and 650 compared to 0.590 and 650). A combination of biomarkers has increased sensitivity to early detection of AKI compared to the application of single biomarkers (17). In a study conducted by Torregrosa et al, the area below the ROC curve for NGAL and KIM- 1 was 0.916 and 0.716 in patients undergoing cardiac surgery, indicating the greater predicting value of NGAL. In their study NGAL levels in urine samples of patients were determined 12 hours after surgery (13). In another similar study, the area below the ROC curve for NGAL and KIM-1 in children referred to the emergency unit was respectively 0.66 and 0.61 , indicating the higher predicting value of NGAL (18). As can be seen, contradictory results have been reported regarding the diagnostic accuracy of NGAL and KIM1. Therefore further studies are needed to compare the diagnostic accuracy of the above two factors at different 
times of sampling as well as their effectiveness.

Despite much efforts conducted to identify AKI the predictive value of biomarkers, the true usefulness of different AKI biomarkers has not yet been fully elucidated. Finding of an ideal biomarker for the early detection of AKI is still under consideration, while several questions concerning the best biomarker/combination of biomarkers, the cutting point with the best sensitivity and specificity, the performance of biomarkers in different populations and different clinical environments, the determination of biomarkers in blood or urine, the best sampling time and the ability to distinguish between functional and structural AKI are existed (16). Our study showed KIM-1 and NGAL biomarkers had a very good diagnostic power in identifying AKI between 24 hours after surgery in patients undergoing $C A B G$ surgery.

\section{Conclusion}

According to our results, patients with AKI had higher levels of serum creatinine, urinary NGAL and KIM-1 compared to non-suspected patients. Serum creatinine had also a significant positive relationship with urinary KIM-1 and NGAL. Urinary KIM-1 and NGAL factors had a high predicting value in AKI detection (the area below the ROC curve was 0.929 and 0.838 , respectively). NGAL had a sensitivity of $86 \%$ and specificity of $80 \%$ at the cutting point of 97.4 and KIM-1 had a sensitivity of $84 \%$ and specificity of $89 \%$ at a cutting point of 14.8 . Generally, it was observed that in patients undergoing CABG surgery urinary KIM-1 and NGAL biomarkers had a proper diagnostic power in identifying AKI between 12-24 hours after surgery. Using these biomarkers can provide faster identification of AKI, which consequently leads to suitable prevention and therapeutic strategies.

\section{Limitations of the study}

The number of patients in the AKI group was relatively small and further studies with a larger population are required. It is suggested that the tests be measured with few hours intervals to determine the best time having the most sensitivity and specificity, which was not possible in our study due to our finance constraints.

\section{Authors' contribution}

MS, SM and AM as the principal investigators of the study participated in the concept and design of the study. MS, FK, FS, NM and SHB were involved in performing study tests and data acquiring. SM and AM revised the manuscript and critically evaluated the intellectual contents. All authors participated in preparing the final draft of the manuscript, revised the manuscript and critically evaluated the intellectual contents. All authors have read and approved the content of the manuscript and confirmed the accuracy or integrity of any part of the work.

\section{Conflicts of interest}

The authors declare that they have no competing interests.

\section{Ethical considerations}

Ethical issues (including plagiarism, data fabrication, double publication) have been completely observed by the authors.

\section{Funding/Support}

The research was the residential thesis of Maryam Saeedi at the department of internal medicine of Shahrekord University of Medical Sciences, which was supported by the university and supported by this university.

\section{References}

1. Chang TI, Leong TK, Boothroyd DB, Hlatky MA, Go AS. Acute kidney injury after CABG versus PCI: an observational study using 2 cohorts. J Am Coll Cardiol. 2014;64:985-94. doi: 10.1016/j.jacc.2014.04.077.

2. Huang T-M, Wu V-C, Young G-H, Lin Y-F, Shiao C-C, Wu $\mathrm{P}-\mathrm{C}$, et al. Preoperative proteinuria predicts adverse renal outcomes after coronary artery bypass grafting. J Am Soc Nephrol. 2011;22:156-63. doi: 10.1681/ASN.2010050553.

3. Maioli M, Toso A, Leoncini M, Gallopin M, Musilli N, Bellandi F. Persistent renal damage after contrast-induced acute kidney injury: incidence, evolution, risk factors, and prognosis. Circulation. 2012;125:3099-107. doi: 10.1161/ CIRCULATIONAHA.111.085290.

4. Tsai TT, Patel UD, Chang TI, Kennedy KF, Masoudi FA, Matheny ME, et al. Contemporary incidence, predictors, and outcomes of acute kidney injury in patients undergoing percutaneous coronary interventions: insights from the NCDR Cath-PCI registry. JACC Cardiovasc Interv. 2014;7:1-9. doi: 10.1016/j.jcin.2013.06.016.

5. Chertow GM, Lazarus JM, Christiansen CL, Cook EF, Hammermeister KE, Grover F, et al. Preoperative renal risk stratification. Circulation. 1997;95:878-84.

6. Boldt J, Wolf $M$. Identification of renal injury in cardiac surgery: the role of kidney-specific proteins. J Cardiothorac Vasc Anesth. 2008;22:122-32. doi: 10.1053/j. jvca.2007.10.008.

7. Rosner MH, Okusa MD. Acute kidney injury associated with cardiac surgery. Clin J Am Soc Nephrol. 2006;1):19-32.

8. Wagener G, Gubitosa G, Wang S, Borregaard N, Kim M, Lee HT. Urinary neutrophil gelatinase-associated lipocalin and acute kidney injury after cardiac surgery. Am J Kidney Dis. 2008;52:425-33. doi: 10.1053/j.ajkd.2008.05.018.

9. Soni SS, Cruz D, Bobek I, Chionh CY, Nalesso F, Lentini P, et al. NGAL: a biomarker of acute kidney injury and other systemic conditions. Int Urol Nephrol. 2010;42:141-50. doi: 10.1007/s11255-009-9608-z.

10. Haase $M$, Devarajan P, Haase-Fielitz A, Bellomo R, Cruz DN, Wagener G, et al. The outcome of neutrophil gelatinase-associated lipocalin-positive subclinical acute kidney injury: a multicenter pooled analysis of prospective studies. J Am Coll Cardiol. 2011;57:1752-61. doi: 10.1016/j. jacc.2010.11.051.

11. Mishra J, Dent C, Tarabishi R, Mitsnefes MM, Ma Q, Kelly 
C, et al. Neutrophil gelatinase-associated lipocalin (NGAL) as a biomarker for acute renal injury after cardiac surgery. Lancet. 2005;365:1231-8.

12. Wagener G, Jan M, Kim M, Mori K, Barasch JM, Sladen RN, et al. Association between increases in urinary neutrophil gelatinase-associated lipocalin and acute renal dysfunction after adult cardiac surgery. Anesthesiology. 2006;105:485.

13. Torregrosa I, Montoliu C, Urios A, Andrés-Costa MJ, Giménez-Garzó C, Juan I, et al. Urinary KIM-1, NGAL and L-FABP for the diagnosis of AKI in patients with acute coronary syndrome or heart failure undergoing coronary angiography. Heart Vessels. 2015;30:703-11. doi: 10.1007/ s00380-014-0538-z.

14. Parikh CR, Thiessen-Philbrook H, Garg AX, Kadiyala D, Shlipak MG, Koyner JL, et al. Performance of kidney injury molecule-1 and liver fatty acid-binding protein and combined biomarkers of AKI after cardiac surgery. Clin J Am Soc Nephrol. 2013;8:1079-88. doi: 10.2215/ CJN.10971012.

15. Zou Z, Zhuang Y, Liu L, Shen B, Xu J, Luo Z, et al. Role of body mass index in acute kidney injury patients after cardiac surgery. Cardiorenal Med. 2018;8:9-17. doi: 10.1159/000477824

16. Khreba NA, Abdelsalam M, Wahab A, Sanad M, Elhelaly R, Adel M, et al. Kidney Injury Molecule 1 (KIM-1) as an Early Predictor for Acute Kidney Injury in Post-Cardiopulmonary Bypass (CPB) in Open Heart Surgery Patients. Int J Nephrol. 2019;2019:6265307. doi: 10.1155/2019/6265307.

17. Han WK, Wagener G, Zhu Y, Wang S, Lee HT. Urinary biomarkers in the early detection of acute kidney injury after cardiac surgery. Clin J Am Soc Nephrol. 2009;4:87382. doi: 10.2215/CJN.04810908.

18. Du Y, Zappitelli M, Mian A, Bennett M, Ma Q, Devarajan P, et al. Urinary biomarkers to detect acute kidney injury in the pediatric emergency center. Pediatr Nephrol. 2011;26:26774. doi: 10.1007/s00467-010-1673-0.

19. Parikh CR, Coca SG, Thiessen-Philbrook H, Shlipak MG, Koyner JL, Wang Z, et al. Postoperative biomarkers predict acute kidney injury and poor outcomes after adult cardiac surgery. J Am Soc Nephrol. 2011;22:1748-57. doi: 10.1681/ ASN.2010121302.

20. Haase M, Bellomo R, Devarajan P, Schlattmann P, HaaseFielitz A; NGAL Meta-analysis Investigator Group. Accuracy of neutrophil gelatinase-associated lipocalin (NGAL) in diagnosis and prognosis in acute kidney injury: a systematic review and meta-analysis. Am J Kidney Dis. 2009;54:1012-24. doi: 10.1053/j.ajkd.2009.07.020.

21. Koyner JL, Bennett MR, Worcester EM, Ma Q, Raman J, Jeevanandam V, et al. Urinary cystatin C as an early biomarker of acute kidney injury following adult cardiothoracic surgery. Kidney Int. 2008;74:1059-69. doi: 10.1038/ki.2008.341

22. Tuladhar SM, Püntmann VO, Soni M, Punjabi PP, Bogle RG. Rapid detection of acute kidney injury by plasma and urinary neutrophil gelatinase-associated lipocalin after cardiopulmonary bypass. J Cardiovasc Pharmacol. 2009;53:261-6. doi: 10.1097/FJC.0b013e31819d6139.

Copyright () 2021 The Author(s); Published by Published by Society of Diabetic Nephropathy Prevention. This is an open-access article distributed under the terms of the Creative Commons Attribution License (http://creativecommons.org/licenses/by/4.0), which permits unrestricted use, distribution, and reproduction in any medium, provided the original work is properly cited. 